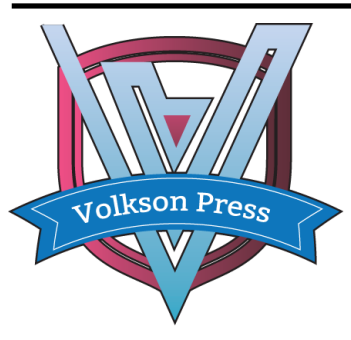

Contents List available at VOLKSON PRESS

New Materials and Intelligent Manufacturing (NMIM)

DOI : http://doi.org/10.26480/icnmim.01.2018.54.57

Journal Homepage: https://topicsonchemeng.org.my/

ISBN: 978-1-948012-12-6

\title{
RESEARCH PROGRESS OF AMINE CATALYSTS FOR POLYURETHANE
}

\author{
Li Ruiduan, Liu Ling, Liu Yanjie, Wang Ben, Yang Jia Jun, Zhang Jibo* \\ Department of Chemical Engineering, Ji Lin Institute of Chemical Technology, No.45 Chengde Street, JiLin City, China. \\ *Corresponding Author Email: zjb@jlict.edu.cn
}

This is an open access article distributed under the Creative Commons Attribution License, which permits unrestricted use, distribution, and reproduction in any medium, provided the original work is properly cited

\section{ARTICLE DETAILS}

\section{Article History:}

Received 26 June 2018

Accepted 2 July 2018

Available online 1 August 2018

\section{ABSTRACT}

\begin{abstract}
Polyurethane products are numerous and widely used in fields, such as foams, elastomers, elastic fibers, coatings, and adhesives, etc. Polyurethane catalyst is an important part of polyurethane. In the production of polyurethane, the catalyst plays an important role. According to the different uses of polyurethane, different catalysts are needed to give polyurethane special properties. Therefore, polyurethane has many types of catalys. But, it is used to polyurethane foaming and curing is mainly amine catalysts. Amine catalysts and their main technologies are mainly introduced in this paper.
\end{abstract}

\section{KEYWORDS}

Amine catalysts, Polyurethane, foams

\section{INTRODUCTION}

Polyurethane products are numerous and widely used in fields, such as foams, elastomers, elastic fibers, coatings, and adhesives, etc. After years of development, China has become the world's largest producer and consumer of polyurethanes in 2015. The catalyst plays an important role in the production of polyurethanes. Catalyst is a catalyst for many chemical reactions, and it is no exception for polyurethanes. It can shorten the reaction time, improve the production efficiency, promote positive reactions and suppress side reactions. The excellent performance of the catalyst can effectively increase the conversion and selectivity of the reaction, thereby increasing the production of polyurethane. In the production of many polyurethane products, the catalyst is a commonly used additive, and the amount is small and has a great effect.

Polyurethane foams are an important category of polyurethane synthetic materials, and their total output accounts for about $60 \%$ of polyurethane materials. Polyurethane foam is one of the fastest-growing varieties in the modern plastics industry. It is characterized by changing the structure of the chain links in the molecule to enable a wide range of modifications, and in the case of changing the technological formulae, it is obtained from soft to hard foamed products with different densities and properties. Coupled with its shape simply, It is widely used in a variety of fields including insulation, vibration, noise, light weight structural parts, cushions, packaging, automotive interior parts, etc.

\subsection{Polyurethane Preparation Principle}

\subsubsection{Polyester synthesis reaction type}

Adipic acid and diethylene glycol are heated and dehydrated to produce polyester under the protection of nitrogen. As shown by the reaction formula1-1.

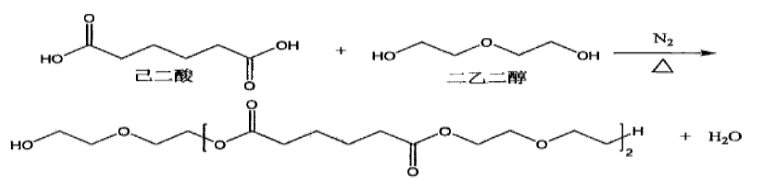

\subsubsection{Preparation of polyurethane foams}

(a) Gradual addition reaction

Polyisocyanates react with polyhydric alcohols (polyoxime, polywaw, or other polyols) to produce polyurethanes. As shown by the reaction formula1-2.

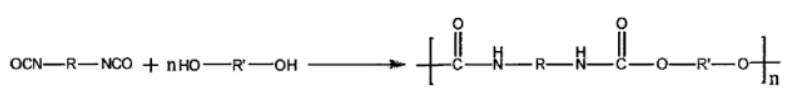

(b) Foaming reaction

Isocyanate and water react to form unstable carbamic acid, then carbamic acid decomposes to amine and $\mathrm{CO}_{2}$. As shown by the reaction formula1-3.

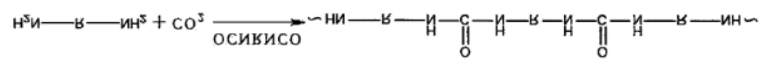

$$
\begin{aligned}
& \mathrm{OCn}-\mathrm{r}-\mathrm{nCO}+\mathrm{H}^{\mathrm{s} O} \longrightarrow[\mathrm{HO}-\mathrm{C}-\mathrm{n}-\mathrm{B}-\mathrm{H}-\mathrm{C}-\mathrm{OH}] \longrightarrow
\end{aligned}
$$

(c) Cross-linking reaction

The ammonia on the nitrogen atom in the carbamate group reacts with the isoacyl acetic acid to form an adenosyl methyl ester, and then the oxygen on the nitrogen atom in the oxime group reacts with the isocyanate to form a constricted vein.

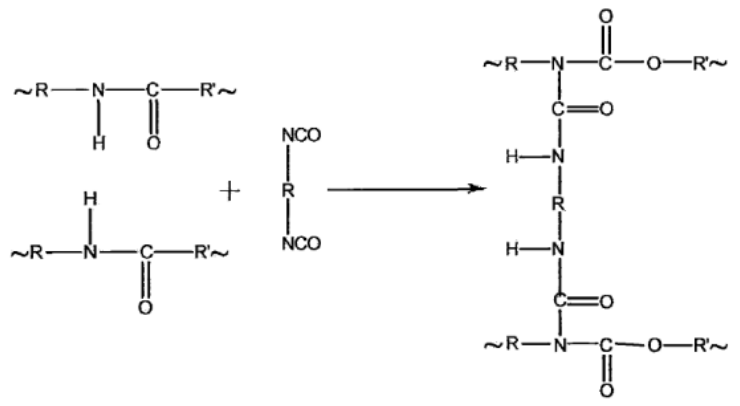


The reaction of -NCO with water in the synthesis of polyurethanes, especially polyurethane foams (Formula (1-3)), and the reaction of -NCO with hydroxyl-terminated polyesters, polyether polyols (formula (1-2)), It is the two most important reactions, in which the tertiary amine catalysts have a strong catalytic effect on them, especially the catalysis of the reaction between - $\mathrm{NCO}$ and $-\mathrm{OH}$ is more clear and wet. The former can promote -NCO reaction with water and quickly produce carbon dioxide gas, so that the polymer volume quickly expands and expands. The latter can promote the rapid growth of polymer molecular chains, the viscosity increases rapidly, and the foam network skeleton strength increases rapidly.

\section{POLYURETHANE CATALYST}

In the process of synthesizing polyurethane, the polyurethane catalyst can increase the reaction rate, increase the production efficiency, and promote the positive reaction and suppress the side reaction selectively. In the production of many polyurethane products, the catalyst is a commonly used additive, although the amount is small, but the role is very great.

Polyurethane catalysts mainly include inorganic salt compounds, organophosphorus compounds, amine catalysts, and organometallic compounds, etc. Although a small amount of inorganic salt compounds and organic phosphonium compounds can be used as a catalyst for polyurethanes, catalysts commonly used in the synthesis of polyurethanes and their raw materials are mainly amine catalysts and organic metal compounds.

With the rapid economic development, people are increasingly demanding for the environment. For the polyurethane industry, the environmental issues include production and use, such as the early use of TDI to gradually shift to a bubble rich in MDI systems, and improve the production environment for production workers. In consumer applications, polyether polyols, amine catalysts, silicone surfactants, flame retardants, and antioxidants all determine the performance of the final product, including VOC emissions such as aldehydes and benzenes. Low-VOC, Low-Fogging, and Low-Odor polyurethane products have been increasingly used in the automotive interior industry. Conventional amine catalysts are currently the focus of attention, while the new generation of amine catalysts can balance the reaction of gels and foams with a wide range of processing technologies. They can meet the low amine emission, low fogging and odor reduction in the polyurethane industry., including all MDI, TDI/MDI and TDI based formulations.

For many years, tertiary amine catalysts are an important part of typical polyurethane foam formulations. Some of the catalysts are biased towards the reaction of water and isocyanate (blowing reaction), while others are biased towards the reaction of polyols with isocyanates (gels reaction), depending on the molecular structure of the catalyst itself and its steric, electronic effects, etc., will affect its function. This work focuses on amine catalysts and their production technology.

\section{FUNCTION OF AMINE CATALYST}

According to different uses of polyurethanes, different catalysts are needed to give polyurethanes special properties. Therefore, there are many types of catalysts for polyurethanes, but the catalysts used for polyurethane foaming and curing are mainly amine catalysts. Polyurethane foam products are mainly synthesized from polyether's, isocyanates, foaming agents, catalysts. Foams are generated in polyurethane reaction systems by physical and chemical methods. The main foaming method is that water and isocyanate are used to produce carbon dioxide to form foam. Amine catalysts are good catalysts for the reaction of NCO and water. That is amine catalysts can promote the foaming reaction. The organometallic catalyst has high selectivity for the reaction of $\mathrm{NCO}$ and $\mathrm{OH}$, but it is insensitive to water and $\mathrm{NCO}$ reactions. The catalytic effect of amines on the polymerization of polyurethanes is accelerated by the formation of $\mathrm{R}-\mathrm{N}=\mathrm{C}=\mathrm{O}$ from the nitrogen atom of the amine and the isocyanate, so that the activation energy of the isocyanate is greatly reduced.

Since the polymerization of polyurethane is a stepwise polyaddition reaction, the hydrogen in the reaction process is transferred from the hydroxyl group (polyester or polyether) to the nitrogen atom of the isocyanate, so that the carbon-nitrogen double bond is opened to form a carbon cation. Carbonium ions are ionically reacted with a hydroxyldepleted hydroxy compound (polyester or polyether) to form polyurethane.

This type of common amine is not considered if the emission of volatile VOCs from the foam, the discoloration, fog, and odor of the PVC skin are not considered. The use of catalysts will continue. These problems have been greatly improved in silicone oils, polyether polyols, flame retardants, and antioxidants. Especially in the fields of furniture, automotive interiors and other areas, this is a key enabler of this technology improvement. The conventional tertiary amine catalysts TEDA, BDMAEE clearly show the release of VOC species in the foam product. The use of large molecular weight tertiary amine catalysts has three distinct advantages in use: First, the molecules of the catalyst are limited in the combined polyether and cannot diffuse themselves, thereby improving odor, PVC skin discoloration and fogging values; secondly, Due to the large molecular weight catalyst, the low vapor pressure drops the volatility at a certain temperature; a third advantage is that the alternative end of the catalyst not only affects the boiling point or vapor pressure of the catalyst, but also reduces its own toxicity. However, compared with the reaction type catalyst, the reaction activity is poor, the amount is large, the process tolerance is narrow, and it has a great impact on the cost.

The catalytic effect of amines on the polymerization of polyurethanes is to accelerate the reaction by the formation of $-\mathrm{R}-\mathrm{N}=\mathrm{C}=\mathrm{O}$ from the nitrogen atom of the amine and isocyanate, so that the activation energy of the isocyanate is greatly reduced, and thus the catalytic activity of the amine compound. It is proportional to the density (alkaline) of the electron cloud on the nitrogen atom and inversely proportional to the volume (space effect) of the radical that the nitrogen atom is attached to [1].

Since the polymerization reaction of polyurethane is a stepwise polyaddition reaction, the ammonia in the reaction process passes through the nitrogen atom of the acetoacid vinegar, so that the carbonnitrogen double bond is opened to form a carbon cation and loses the hydroxy compound of ammonia. The ionic reaction combines to form polyurethane. The reduction of the activation energy of isocyanate does not necessarily accelerate the polymerization reaction. It is only when the activation energy of isocyanate is reduced, and the hydroxy compound is activated to form ammonium ions. Therefore, the polymerization reaction can be accelerated, so the stronger the amine-hydroxy complexing ability, the faster the polymerization reaction proceeds. In general, the change rule of the catalytic activity of the polymerization reaction is: tertiary amine $>$ secondary amine $>$ primary amine.

\section{THE MAIN TYPES OF AMINE CATALYSTS AND THEIR SYNTHESIS TECHNIQUES}

For polyurethanes, especially two main reactions of polyurethane foam synthesis $\quad$-NCO with water and -NCO with a hydroxyl terminated polyester or polyether polyol, tertiary amine catalysts have a strong catalytic effect, especially the catalytic effect of $-\mathrm{NCO}$ and $-\mathrm{OH}$ is more pronounced. The former can promote the growth of polymer molecular chains, the increase in viscosity and the increase of the foam network strength rapidly. The latter can promote the reaction of -NCO with water, and quickly produce carbon dioxide gas, so that the polymer volume increases rapidly and expands. There are many varieties of tertiary amines catalysts of polyurethanes. According to their chemical structures, they can be basically divided into fatty amines, alicyclic amines, aromatic amines, alcohol amines and their ammonium compounds.

\subsection{Aliphatic amine catalysts}

Aliphatic amine catalysts include N,N-dimethylcyclohexane, triethylenediamine, N,N,N,N-tetramethylalkylenediamine, N,N,N,Npentamethyldiethylenetriamine, riethylamine, $\mathrm{N}, \mathrm{N}$-dimethylbenzylamine, $\mathrm{N}, \mathrm{N}$-dimethylhexadecylamine, $\mathrm{N}, \mathrm{N}$-dimethylbutylamine, etc.

In the early 1950s, American Air Products Company was involved in the development of triethylenediamine, and in conjunction with the 
development of the polyurethane industry, this type of catalyst was launched. The registered trademark was DABCO, which laid the foundation for the application of tertiary amine catalysts in polyurethane industry. It has also been found that the tertiary amine catalysts act synergistically. For example, the use of mixed catalytic system of dimethylethanolamine and triethylenediamine in the production of rigid polyurethane foams can effectively improve the catalytic effect of the reaction, shorten the aging time of the foam products, and improve the production efficiency.

In order to improve the economics and environmental friendliness of $\mathrm{N}, \mathrm{N}-$ dimethyl n-butylamine synthesis method, Zhang used a route of catalytic amination with n-butylamine and methanol as the main raw materials, and the atomic utilization ratio was high. The effects of the active components of the catalyst and reaction conditions on the synthesis of $\mathrm{N}, \mathrm{N}$-dimethyln-butylamine were investigated [2].

Aliphatic Isocyanate Polyurethane Catalyst is a gel-type polyurethane catalyst. Compared with traditional catalysts, it has better solubility in polyurethane systems, shorter gel time and tack-free time, faster curing speed, etc. It is suitable for polyurethane spray foam, coatings and adhesives.

The dehydration of hydroxyethylamine compounds to triethylenediamine is the focus of current research. This process uses ethanolamine or diethanolamine, triethanolamine, etc. as raw materials, in the presence of a catalyst (such as ZSM-5 or TS-1), and ammonia. Such reactions, condensation dehydration, to obtain the product triethylenediamine. The reaction process is shown in Figure 1. Triethylenediamine has a unique cage structure in which two nitrogen atoms are directly linked to three ethylene groups to form a bimolecular structure, which is very dense and symmetrical. Since there are not only other substituents on the $\mathrm{N}$ atom that increase the steric hindrance, but also have a pair of very accessible empty electrons, in the catalytic foaming system, after the urethane bond is formed, the triethylenediamine will dissociate and participate in the next catalytic process. Thus, although triethylenediamine is not a strong base, it exhibits extremely high catalytic activity for the reaction of isocyanate groups and active hydrogen compounds [3]. The reaction process is shown in Figure 1.

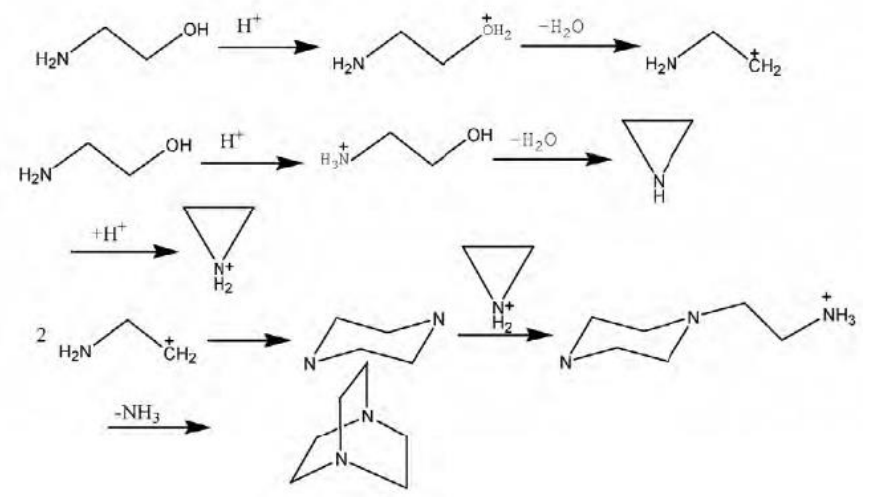

Figure 1: The synthetic route of DABCO

\subsection{Alicyclic amines catalysts}

Alicyclic amines include triethylenediamine, N-ethylmorpholine, Nmethylmorpholine, N, N-diethylpiperazine, and N, -diethyl-2-methyl Piperazine, N,N-bis-( $\alpha$-hydroxypropyl)-2-methylpiperazine, $\quad \mathrm{N}$ hydroxypropyldimethylmorpholine, etc. Ajai synthesized N-\{2-(4methoxyphenyltelluro) ethyl $\}$ morpholine and bis $\{2$-(N-morpholino) ethyl\} telluride, respectively, which are first tellurated derivatives of morpholine [4]. Yang synthesized piperazine using ethylene glycol as a raw material, with amination technology in a fixed reactor [5]. Pan synthesized an asymmetrically structured diamine-organic amine 1-(2Hydroxyethyl)-4-(2-Hydroxypropyl) Piperazine [6].

\subsection{Alcohol amines catalysts}

Alcohol amines catalysts have less catalytic activity than ether amines and polyamines and higher additions. However, alcohol amine catalysts have lower prices. Under certain conditions, adding appropriate amounts of alcohol amines can be added to adjust the reaction rate and reduce costs. It can also be used in certain foams alone. Alkanol amines are also important raw materials for the synthesis of other amine catalysts. Alcohol amine catalysts include triethanolamine, $\mathrm{N}, \mathrm{N}$-dimethylethanol amine, etc. Alcohols amine are a type of reactive catalyst that can be used in conjunction with other high activity catalysts.

For comfortable driving conditions, flexible polyurethane foams are used for sound absorbers in noise, vibration, harshness systems. The cellular structures of polyurethane foams are important to improve the sound absorption efficiency, and the cell morphologyis strongly dependent the use of experimental ingredients such as catalysts [7]. Wang used an alcohol containing an amino group to react with a cyclic carbonate to prepare a urethane group-containing diol and then reacted with methacrylic anhydride to synthesize a reactive diluent having a nonisocyanate structure [8]. This shows that the reactive diluent can significantly improve the impact resistance and elongation at break of the coating film relative to the conventional ethylene glycol dimethacrylate.

\subsection{Aromatic amine catalysts}

The commonly used aromatic amines for polyurethane catalysts are pyridine, N,N-dimethylpyridine, etc. Most of the synthesis of azidopyridines is in the 2,4,6 point of the pyridine ring [9]. The synthesis of 3,5-Dimethoxycarbonylamino-pyridine faces serious problems such as serious pollution, complicated operation, harsh conditions, and expensive catalysts [10]. Hu synthesied of 3,5-diaminopyridine and 3,5dimethoxycarbonylpyridine using 3,5-dimethylpyridine as a raw material through oxidation and amidation [11].

$\mathrm{N}, \mathrm{N}$-Dimethylaniline was synthesized using the dimethyl carbonate and aniline as starting materials. The effects of reaction temperature, reaction pressure, reaction time, the molar ratio of the $\mathrm{N}, \mathrm{N}$-dimethylaniline. With dimethyl carbonate on yield was investigated. The results indicate that the $\mathrm{N}, \mathrm{N}$-dimethylaniline yield can reach $90 \%$ when the reaction temperature was $200^{\circ} \mathrm{C}$, the reaction pressure was $3.5 \mathrm{MPa}$, the reaction time was $2 \mathrm{~h}$, the $n($ aniline):n(dimethyl cabonate) was 1:1.25 [12]. The reaction process is shown in Figure 2.

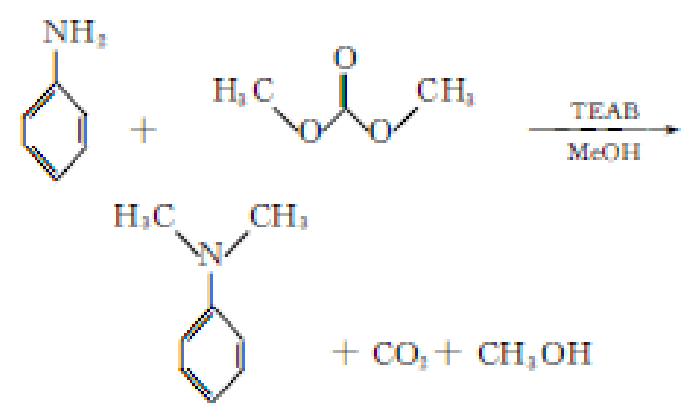

Figure 2: The synthetic route of N, N-Dimethylaniline

\subsection{Ether amine catalysts}

The 2,2'-dichloroethyl ether (abbreviated as chloroether) amination method is an earlier reported BDMAEE synthesis process. In 1968, Fedor et al. used chloroether as a raw material to synthesize BDMAEE through ammoniation and demethylation [13]. The reaction process is shown in Figure 3.

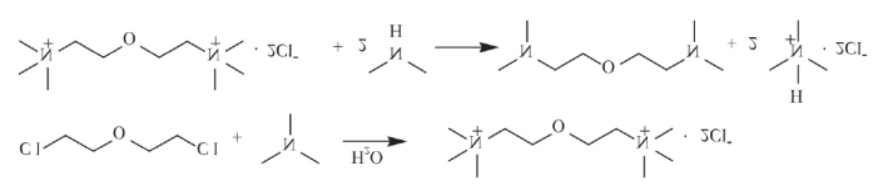

Figure 3: The synthetic route of BDMAEE by amination of chloride ester 
In 1997, Kordas et al. reported the preparation of BDMAEE by the condensation of formaldehyde and 2,2'-diaminoethyl ether [14]. The reaction process is shown in Figure 4.

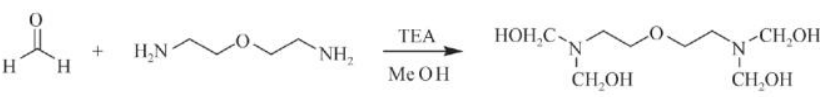

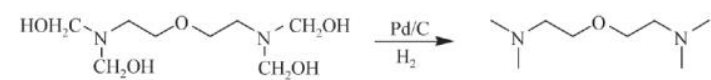

Figure 4: The synthetic route of BDMAEE by condensation of amido ester and formaldehyde

In 2013, Chen Songlin and Huang Daqi et al. reported on a two-step process for the synthesis of BDMAEE using dimethylaminoethoxyethanol (abbreviated as DMAEE) through aminolysis and condensation reduction [15]. The reaction process is shown in Figure 5.

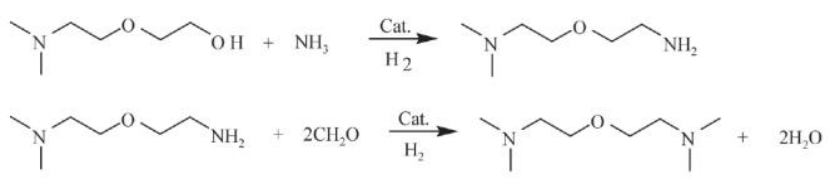

Figure 5: The synthetic route of BDMAEE by ammonolysis and reduction of DMAEE

In high-rebound soft foams, bis(2-dimethylaminoethyl) ether) BDMAEE is used as a conventional foaming catalyst due to its unique chemical structure, which is a typical strong and efficient tertiary amine catalyst, promotes the reaction of water with isocyanate, also known as the foaming reaction or early reaction. The vast majority of foam formulations use BDMAEE in combination with triethylenediamine TEDA. TEDA is a commonly used gel catalyst. Currently, there are many commercially available catalysts that use a mixture of catalysts for the purpose of balancing effects and process rates.

The ether amines with $\mathrm{N}, \mathrm{N}$-dialkylamino oxime ether structures are broad-spectrum catalysts for polyurethane foams. It has catalytic activity for both polyurethane foaming and gelling reactions, especially for the foaming reaction, has high sensitivity for adjusting and controlling the foaming and gelling reaction, and has a synergistic effect with organotin catalysts than triethylenediamine. When an ether amine catalyst is used, the organotin catalyst can be used in a wide range of adjustability, foaming ability is enhanced, the effective gas utilization rate is high, the generated urea concentration is high, and the hardness and height of the foam are increased. The role of ether amines in soft foams is getting more and more noticeable, and ether amine synthesis technology has developed rapidly. Yu describes in detail the use of phosphazene catalysts in the production of polyether polyols and also outlines the use of polyether polyols catalysts in the preparation of polyurethane foams [16].

\section{CONCLUSION}

Various types of new catalysts have been introduced abroad. The gap between China and other country is very large. Due to the foreign technology blockade, insufficient supply of domestic basic raw materials, slow progress in the development of new products, a single variety of amine catalysts, insufficient quantities, and long-term inability to selfsufficient, most Polyurethane catalysts rely on imports. Although triethylenediamine and dimethylethanolamine have been produced, but the production scale is small, quality is unstable and produced cost is high, it cannot meet the domestic market. The research and development of domestic amine catalysts aims at replacing imported products, and studies new processes that are easy to use for production expansion and have potential for production.

\section{REFERENCES}

[1] Zhili, R., Hongsheng, Z., Haiqin, Y. 1985. Comparison of the activity of amine catalysts in polyurethane polymerization [J]. Polyurethane industry, 3, 1-6.

[2] Xu, Z., Tao, Z., Xiao-xiang, C. 2009. Synthesis of N, N-dimethyl-nbutylamine with tubular reactor [J]. Journal of Zhejiang University (Engineering Science), 43 (09), 1684-1686+1738.

[3] Huijian, S., Liwei, W., Hui, L. 2014. Titanium silica molecular sieve catalytic ethanolamine synthesis of triethylenediamine technology research [J]. Modern Chemical Industry, 34 (2), 110-114.

[4] Ajai, K., Singh, J., Sooriyakumar, S. 2000. N $\quad-\{2-(4-$ Methoxyphenyltelluro) ethyl $\}$ morpholine (L 1) and bis $\{2-(\mathrm{N}$ morpholino) ethyl telluride (L 2): synthesis and complexation with palladium (II) and mercury (II). Crystal structures of trans - [PdCl 2 (L 1) 2] and trans- [PdCl 2 (L 2) 2] [J]. Journal of Organometallic Chemistry, 612 (1).

[5] Zhen, Y., Feixiang, G., Yonghong, Q. 2016. Catalyst and Amination Process for the Synthesis of Piperazine from Ethylene Glycol [J]. Chemical Reaction Engineering and Technology, 32 (06), 570-575.

[6] Fei, P., Xiao-hong, C., Cheng-wu, S. 2016. Synthesis and Characterization of 1-(2-Hydroxyethyl)-4-(2-Hydroxypropyl) Piperazine [J]. Journal of Chemical Engineering of Chinese Universities, 30 (01), 142-148.

[7] Kim, S.K., Sung, G., Gwon, J.G. 2016. Controlled phase separation in flexible polyurethane foams with diethanolamine cross-linker for improved sound absorption efficiency [J]. International Journal of Precision Engineering and Manufacturing-Green Technology, 3 (4), 367373.

[8] Wang, X.J., Soucek, M.D. 2013. Investigation of non-isocyanate urethane dimethacrylate reactive diluents for UV-curable polyurethane coatings [J]. Progress in Organic Coatings, 76, 1057-1067.

[9] Yuanyuan, Y., Guoquan, Z., Xinzhi, C. 2004. An Improved Synthesis of 4Aminopyridine [J]. Chinese Journal of Applied Chemistry, 21 (5), 530-531.

[10]Zhou, Y.F., Gregor, V.E., Ayida, B.K. 2007. Synthesis and SAR of 3, 5Diamino-piperidine Derivatives: Novel Antibacterial Translation Inhibitors as Amino-glycoside Mimetics [J]. Bioorganic \& Medicinal Chemistry Letters, 17, 1204-1208.

[11] Bingcheng, H., Changyu, L., Ming, L. 2012. A New Synthetic Route to 3, 5-Diaminopyridine and 3, 5-Dimethoxycarbonylamino-pyridine [J]. Chinese Journal of Applied Chemistry, 29 (01), 80-83.

[12] Zhengling, W., Peiyu, G., Yanbin, W. 2016. Synthesis process conditions of N,N-dimethylaniline [J]. Fine and Specialty Chemicals, 24 (09), 39-41.

[13] Poppelsdorf, F., Charleston. 1964. Production of Bis [beta (N, Ndimethylamino ) -alkyl] Ethers: US, 3400157A[P]-11-27

[14] Imre, K., Karoly, S., Attila, G. 1997. Process for the Preparation of Nmethylated Amines by Metal-catalyzed reductive methylation: EU, 219183 B[P]. 07-15.

[15] Songlin, C., Daqi, H. 2013. Preparation of bis(2-dimethylaminoethyl) ether: China, CN10345-35A[P]. 12-18.

[16] Jiankun, Y., Yunduo, C. 2012. Novel Polyether Polyols Synthesized by Using Phosphazene Catalysts and New [J]. Chemical Propellants \& Polymeric Materials, 10 (02), 1-7. 